\title{
$A$-Site Ordering versus Electronic Inhomogeneity in Colossally Magnetoresistive Manganite Films
}

\author{
V. Moshnyaga, ${ }^{1}$ L. Sudheendra, ${ }^{1}$ O. I. Lebedev, ${ }^{2}$ S. A. Köster,${ }^{1}$ K. Gehrke, ${ }^{1}$ O. Shapoval,${ }^{3}$ A. Belenchuk, ${ }^{3}$ B. Damaschke, ${ }^{1}$ \\ G. van Tendeloo, ${ }^{2}$ and K. Samwer ${ }^{1}$ \\ ${ }^{1}$ Erstes Physikalisches Institut, Universität Göttingen, Friedrich-Hund-Platz 1, D-37077 Göttingen, Germany \\ ${ }^{2}$ EMAT, University of Antwerp, Groenenborgerlaan 171, B-2020 Antwerpen, Belgium \\ ${ }^{3}$ Institute of Applied Physics, Strada Academiei 5, MD-2028, Chisinau, Moldova
}

(Received 15 December 2005; published 8 September 2006)

\begin{abstract}
Epitaxial $\mathrm{La}_{3 / 4} \mathrm{Ca}_{1 / 4} \mathrm{MnO}_{3} / \mathrm{MgO}(100)$ (LCMO) thin film shows an unusual rhombohedral $(R-3 c)$ structure with a new perovskite superstructure at room temperature due to the CE-type ordering of La and $\mathrm{Ca}$ with modulation vector $q=1 / 4[011]$. A-site ordered film was found to be electronically homogeneous down to the $1 \mathrm{~nm}$ scale as revealed by scanning tunnelling microscopy/spectroscopy. In contrast, orthorhombic and $A$-site disordered LCMO demonstrate a mesoscopic phase separation far below the Curie temperature $\left(T_{C}\right)$. Unique $\mathrm{La} / \mathrm{Ca}$ ordering compensates the cation mismatch stress within one supercell, $a_{S} \approx 1.55 \mathrm{~nm}$, and enhances the electronic homogeneity. The phase separation does not seem to be a unique mechanism for the colossal magnetoresistance (CMR) as very large CMR $\approx 500 \%$ was also observed in $A$-site ordered films.
\end{abstract}

DOI: 10.1103/PhysRevLett.97.107205

PACS numbers: 75.47.Lx, 68.37.- d, 71.30.+h

It is commonly believed that the basic physics of colossal magnetoresistance (CMR) [1] perovskite manganites, $\mathrm{Ln}_{1-x} \mathrm{Ae}_{x} \mathrm{MnO}_{3}$ (where $\mathrm{Ln}^{3+}=\mathrm{La}, \mathrm{Nd}, \mathrm{Sm}$, etc., and $\mathrm{Ae}^{2+}=\mathrm{Ca}, \mathrm{Sr}$, and $\mathrm{Ba}$ are lanthanide and alkaline earth ions, respectively) is governed by the interplay between spin , charge, and lattice degrees of freedom. The metalinsulator (MI) transition temperature, $T_{\mathrm{MI}}$, and $T_{C}$ is controlled by $A$-site substitutions in the perovskite lattice with corresponding changing of the average ionic radius of the $A$-site cation, $\left\langle r_{A}\right\rangle$, and the tolerance factor, $t=\left(r_{A}+\right.$ $\left.r_{O}\right) / \sqrt{2}\left(r_{B}+r_{O}\right)[2-4]$. A-site occupancy in manganites has a random distribution of $A$-site cations similar to a solid solution. In the case of optimally doped $(0.2<x<0.4)$ CMR manganites [5], the disorder not only reduces $T_{C}$ [5] due to size mismatch strain fields and the corresponding Jahn-Teller (JT) distortions of the $\mathrm{MnO}_{6}$ octahedra [6] but also causes electronic inhomogeneity or phase separation (PS) [7-10]. PS has been modeled both for cation disorder [11] and stress [12]. The CMR effect was attributed to the disorder induced PS, imposed on the first order phase transition [13]. A-site ordering by ( $\mathrm{Ln}-\mathrm{O}) /(\mathrm{Ba}-\mathrm{O})$-layering along the $c$ axis, similar to superconducting cuprates, was reported for "half-doped" manganites, $\mathrm{Ln}_{1 / 2} \mathrm{Ba}_{1 / 2} \mathrm{MnO}_{3}$ [14-16]. To the best of our knowledge there are no reports on $A$-site ordered and optimally doped CMR manganites, neither for bulk nor for thin films. $\mathrm{La}_{3 / 4} \mathrm{Ca}_{1 / 4} \mathrm{MnO}_{3} / \mathrm{MgO}(100)$ (LCMO) with relatively small average radius of $A$-site cation, $\left\langle r_{A}\right\rangle=1.205 \AA$, and tolerance factor, $t=0.97<1$, exhibits the orthorhombic $\left(P_{\text {nma }}\right)$ structure at room temperature and transforms to a rhombohedral $(R-3 c)$ structure at $700 \mathrm{~K}$ [6]. In this Letter we report the existence of epitaxial LCMO film with rhombohedral $(R-3 c)$ structure and a new type of perovskite superstructure due to $\mathrm{La} / \mathrm{Ca}$ ordering. We show that such an ordering at the $A$-site positions not only brings about long range Coulomb order, but also suppresses cation mismatch strain within the superstructure unit cell.

LCMO films with nominal Ca-doping level $x=1 / 4$ were epitaxially grown on $\mathrm{MgO}(100)$ substrates by a metalorganic aerosol deposition (MAD) technique [17,18]. The thickness of the films is $d=80-90 \mathrm{~nm}$ as determined from small angle $x$-ray scattering. X-ray diffraction (XRD) analysis shows "cube-on-cube" epitaxy of LCMO on $\mathrm{MgO}(100)$ and indicates a stress-free state of the film: the film lattice parameter, $a_{p}=0.3875-0.3872 \mathrm{~nm}$, is very close to the bulk value [19]. Remarkably, in LCMO films $(R-3 c)$ structure was observed by transmission electron microscopy (TEM) and electron diffraction (ED) analysis, shown in Fig. 1. The brighter basic reflections in both patterns show a square arrangement with $a \approx a_{p}$. Weak satellite reflections on both ED patterns suggest a modulated structure with a modulation vector $q_{1} \approx \frac{1}{4} \times$ $[011]_{C}^{*}$ in the cross section [Fig. 1(c)] and $q_{2} \approx \frac{1}{2}[001]_{C}^{*}$ in the plain-view ED pattern [Fig. 1(a)]. High resolution (HREM) plane-view and cross-section images are shown in Fig. 1(b) and 1(d), respectively. In the plane view a twinned structure is observed; therefore the modulation in the ED patterns is only one dimensional, but appears in two symmetry related directions. The $(\mathrm{La}, \mathrm{Ca}) \mathrm{O}$ layers [indicated by arrows in Fig. 1(b) and 1(d)] are imaged as rows of brighter dots, whereas La-O layers are imaged as rows of less bright dots in Fig. 1(b). The cross-section HREM image in Fig. 1(d) gives even more evidence for this modulated structure. The Fourier transform of this modulated area exhibits a pattern very similar to the corresponding ED pattern in Fig. 1(c). The modulation shows up as alternative blocks of $2 a_{p} \times a_{p}$ size of a different brightness, indicating an ordering of La and Ca columns. In 


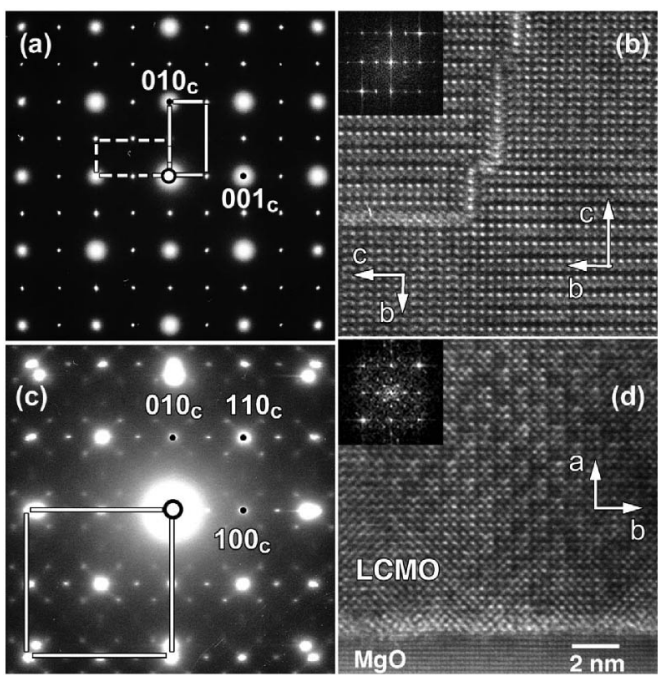

FIG. 1. Plan-view (a) and cross-section (c) electron diffraction patterns of the cation ordered $\mathrm{La}_{3 / 4} \mathrm{Ca}_{1 / 4} \mathrm{MnO}_{3}$ film with modulated structure. The ED pattern obtained from the $\mathrm{MgO}$ substrate indicated by white square in (c). The white rectangles in (a) correspond to the twinned LCMO structure. Plan-view (b) and cross-section (d) HREM images of the same film. The Fourier transforms from single domain of the modulated area [insets in (b) and (d)] exhibit a pattern similar to the corresponding ED patterns in (a) and (c).

Fig. 2 a model for the $\mathrm{La} / \mathrm{Ca}$ ordering is shown, which is in agreement with the measured ED and HREM. The La-Ca ordering involves an alternating stacking of $\mathrm{La}-\mathrm{O}$ and (La,Ca)-O layers along the $[010]_{C}$ direction. The $\mathrm{La}$ and $\mathrm{Ca}$ are ordered within the $\mathrm{La}(\mathrm{Ca})-\mathrm{O}$ layer along the $[100]_{C}$ direction: two La atoms alternate with two $\mathrm{Ca}$ atoms [see the $[001]_{C}$ view in Fig. 2(b)]. The layering of La-O and (La,Ca)-O planes becomes evident along the $[011]_{C} \mathrm{~d}-$ irection. In this direction the film can be viewed as composed of two repeating La-Mn-O (LMO) layers followed by the two layers of La-Mn-Ca-Mn-O (LMCMO). Both layers together form the body- centered cubic superstructure unit cell. The theoretical cation stoichiometry, $\mathrm{La}_{3 / 4} \mathrm{Ca}_{1 / 4} \mathrm{MnO}_{3}$, agrees with the nominal composition. The simulated ED patterns in Fig. 2 correlate very well with the measured ones (Fig. 1). The image simulations [the insets in Fig. 2(a) and 2(b), ] based on the proposed model, are also in agreement with the experimental images.

We also looked for PS in cation ordered LCMO ( $O$ film) in comparison with $A$-site disordered orthorhombic LCMO ( $D$ film) of the same composition, $x=1 / 4$, using scanning tunneling microscopy/spectroscopy (STM/STS) [9]. The surface of the $O$ film [Fig. 3(a)] looks very smooth and flat with an average surface roughness, RMS $=0.2 \mathrm{~nm}$. One-unit cell height steps are clearly seen in between $100-$ $200 \mathrm{~nm}$ wide terraces. The $D$ film [Fig. 3(b)] shows crystalline grains with diameter $\sim 50 \mathrm{~nm}$ and RMS $=2 \mathrm{~nm}$. In Fig. 3(c) and 3(d) we present the distribution of the local tunneling conductance, $\sigma=(d I / d V)_{V=0}$, measured in the ferromagnetic state for $T=115 \mathrm{~K} \ll T_{C}$ for the $O$ and $D$

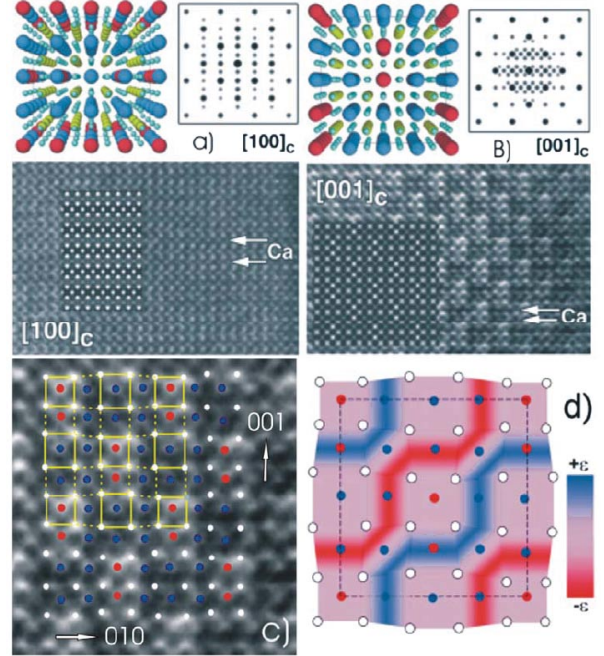

FIG. 2 (color). Structural model of the La-Ca ordered $\mathrm{La}_{3 / 4} \mathrm{Ca}_{1 / 4} \mathrm{MnO}_{3}$ phase, taking into account the experimental evidence: (a) represents the projection along the $[100]_{C}$ direction and (b) represents the projection along the $[100]_{C}$ direction. The corresponding calculated ED patterns and simulated HREM images obtained at a defocus value (Af), Af $=-30 \mathrm{~nm}$ and thickness $t=18 \mathrm{~nm}$ based on the proposed model (given as inset in HREM images) show a good agreement with the experimental ones. In (c) a fragment of a HREM image (b) is shown with cations superimposed (red-Ca and blue-La) and Mn (white). The distribution of local stress is shown in (d) with compressivelike (red stripes) and tensilelike (blue stripes). The overall stress is compensated within one supercell, $a_{S}=4 a_{p}=1.55 \mathrm{~nm}$.

films, respectively. The $O$ film [Fig. 3(c)] shows spatially homogenous, at least down to $1 \mathrm{~nm}$ scale (the limit of our STS setup), tunneling conductance of metallic type, i.e., $\sigma \neq 0$. The conductance map of the $D$ film [Fig. 3(d)] is inhomogeneous - insulating regions, $\sigma \approx 0$, of about 5$20 \mathrm{~nm}$ size ["black clouds" in Fig. 3(d)] are intermixed with metallic (red) regions. Thus, even for $T \ll T_{C}$ the $D$ film shows a mesoscopic PS, whereas the $O$ film does not. In Fig. 4(a) we compare the temperature dependence of the resistivity, $\rho(T)$, for the same $O$ and $D$ films studied by STS/STM (Fig. 3). The MI transition occurs at $T_{\mathrm{MI}}=$ $272 \mathrm{~K}$ and $264 \mathrm{~K}$ for the $O$ and $D$ film, respectively. It is characterized by dimensionless derivative of the resistivity, $\alpha_{\rho}=(d \rho / \rho) /(d T / T)$ [20], the maximum of which describes the sharpness of the transition. Such a formulation accounts for different values of resistivity as well as different transition temperatures for $O$ and $D$ LCMO films. As one can see from Fig. 4(a) (inset) the MI transition is very sharp for $O$ film, yielding $\alpha_{\rho \max }=97$, as compared to $D$ film with $\alpha_{\rho \max }=25$. Interestingly, the $O$ film [the inset to Fig. 4(a)] shows large CMR $=100 \% \times[(\rho(0)-\rho(5 T)] /$ $\rho(5 T) \approx 500 \%$ [ $\approx 83 \%$ if normalized to $\rho(0)$ ], which is comparable with that in $D$ film: CMR $=435 \%$ (81\%). The paraferromagnetic transition [Fig. 4(b)], characterized by $\alpha_{M}=(d M / M) /(d T / T)$, is also significantly sharper in the $O$ film $\left(\alpha_{M \min }=-117\right)$ than in the $D$ film $\left(\alpha_{M \text { min }}=\right.$ $-54)$ in close agreement with the observed electronic 


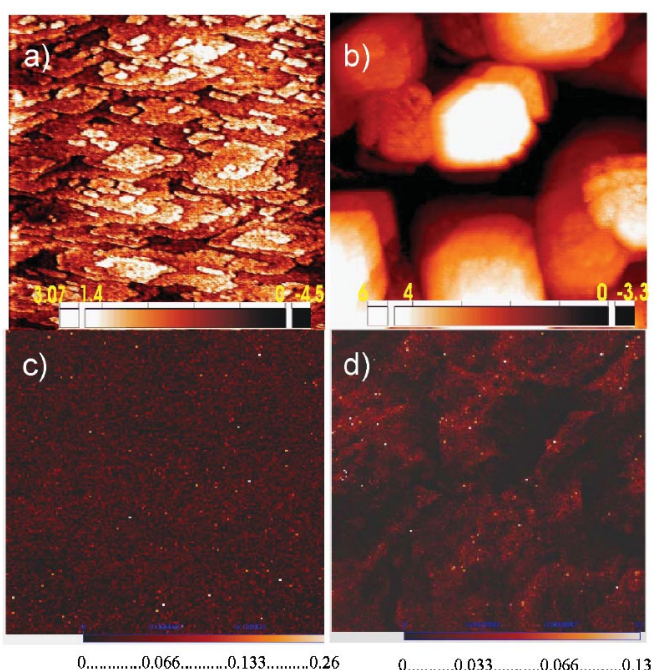

FIG. 3 (color). Scanning tunneling microscopy (STM) morphology image $\left(500 \times 500 \mathrm{~nm}^{2}\right)$ of a pseudocubic LCMO $(O$ film) (a) and $250 \times 250 \mathrm{~nm}^{2}$ STM morphology scan of the orthorhombic $D$ film (b). The scale bar refers to $\mathrm{nm}$. The tunneling conductivity map of $O$ film (c)- and $D$ films (d), obtained from the same regions as in Fig. 3(a) and 3(b), respectively. The electronically homogeneous behavior in the $O$ film and metallike (red) and insulatorlike (black) coexistence in the $D$ film are evident. The scale represents the conductance in $\mathrm{nA} / V$.

homogeneity. For $x \approx 1 / 4$ and 0.3 , a following correlation was established [see Fig. 4(c)]: the larger the $\alpha_{\rho \max }$, the larger the $T_{\mathrm{MI}}$. The largest value was found for the $O$ film $(x \approx 1 / 4)$.

We analyzed the projections of the perovskite unit cells from the cross-section HREM images by assuming the positions for La- (blue), Ca- (red), and Mn- (white) and superpose them on the cross-section HREM in Fig. 2(c). Remarkably, lattice strains due to the $\mathrm{La} / \mathrm{Ca}$ size mismatch, which are crucial for a PS and magnetotransport phenomena, are compensated within a more symmetric $(R-3 c)$ structure with an enlarged superlattice cell $a_{s}=$ $4 a_{p} \approx 1.55 \mathrm{~nm}$. Basic LMO and CMO cells, approximated as quadrangles around $\mathrm{La}(B$ cell $)$ and $\mathrm{Ca}(R$ cell $)$ atoms, respectively, are evidently not equal: their lengths relate exactly to their lattice parameters, i.e., $a_{\mathrm{LMO}}=$ $0.390 \mathrm{~nm}$ and $a_{\mathrm{CMO}}=0.373 \mathrm{~nm}$. This indicates a stressfree state of $R$ and $B$ cells. Along [001 $]_{C}$ and $[010]_{C}$ axes basic cells are linked by the intermediate, $B 1$ cells, which accommodate LMO/CMO local stresses via axial deformation with intervening tensile, $B 1+$, and compressive, $B 1-$, strains of (Mn-Mn) distances, probably due to a change of the (Mn-O-Mn) angle. The sequence of strained domains is the following:
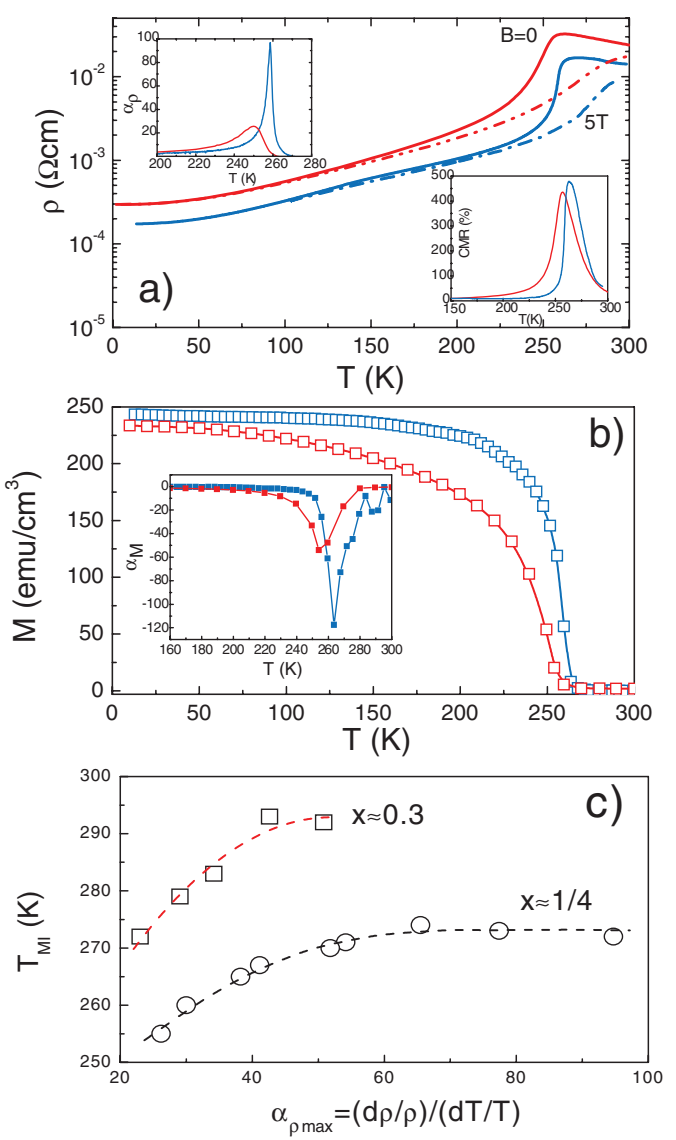

FIG. 4 (color). (a) Temperature dependence of the resistivity, $\rho(T, 0), B=0$ for cation ordered film (solid blue curve) and for an orthorhombic film (solid red curve) of the same $\mathrm{La}_{3 / 4} \mathrm{Ca}_{1 / 4} \mathrm{MnO}_{3}$ composition. The broken lines correspond to the $\rho(T, 5 T)$. The right bottom inset relates to the evaluated temperature dependence of $\mathrm{CMR}=100 \%[R(0)-R(5 T)] /$ $R(5 T)$. The top left inset shows temperature dependence of $\alpha \rho=(d \rho / \rho) /(d T / T)$; (b) Field cooled magnetization as a function of temperature for the same films as in (a), measured in external field $H=100$ Oe. Inset: $\alpha_{M}=(d M / M) /(d T / T)$ function vs temperature. (c) Correlation between the metalinsulator transition temperatures, $T_{\mathrm{MI}}$, for $\mathrm{LCMO} / \mathrm{MgO}$ films with two compositions and disorder, quantified by the function $\alpha_{\rho \max }=(d \rho / \rho) /(d T / T)$. The connecting lines are drawn as guide to the eye.

$\left[R-\left(B 1_{+}\right)-B-\left(B 1_{-}\right)\right]-\left[\left(R-\left(B 1_{+}\right)-B-\left(B 1_{-}\right)\right]-\ldots\right.$.

Along diagonals of the supercell, the local stress has a rather biaxial nature resulting in parallelogramlike deformation, $\left(B 1+_{-}\right)$, with the following sequence of strain domains:

$$
\left[R-\left(B 1_{+-}\right)-R-\left(B 1_{+-}\right)\right]-\left[\left(R-\left(B 1_{+-}\right)-R-\left(B 1_{+-}\right)\right]-\ldots\right.
$$

The resulting distribution of local stresses within one supercell is shown in Fig. 2(d). One can see tensilelike (blue) and the compressivelike (red) stressed perovskite cells arranged in a "zigzag-stripe" fashion along the $[011]_{C}$ diagonal of the supercell. The overall stress, being the sum of red and blue regions, is fully compensated within one supercell. In other 
words, the ordered LCMO film along the [011] direction looks as infinite superlattice, $\left[2^{\prime \prime} \mathrm{LMO}^{\prime \prime} /\right.$ $\left.2^{\prime \prime} \mathrm{La}_{1 / 2} \mathrm{Ca}_{1 / 2} \mathrm{MnO}_{3}^{\prime \prime}\right]_{N}$, in which the lattice misfit $\varepsilon=$ $\left(a_{\mathrm{LMO}}-a_{\mathrm{LCMO}}\right) / a_{\mathrm{LMO}}=1.5 \%$ can be accommodated by the mechanism shown above. Such a layering looks energetically more favorable than a simple $[\mathrm{LMO} / \mathrm{CMO}]_{N}$ layering, which would result in a significantly larger lattice misfit, $\varepsilon=4.5 \%$. We believe that the above stress accommodation drives the formation of the $(R-3 c)$ structure, which diminishes lattice distortions in the $O$ film, yielding a sharp phase transition and homogeneous ferromagnetic (FM) metallic ground state for $T<T_{C}$. In contrast, disorder in the $D$ film $\left(P_{\text {nma }}\right)$ stabilizes PS with nanoscale regions with cooperative JT effect $[21,22]$.

The correlation between the transition temperature and disorder within the same bandwidth $(x \approx 1 / 4$ and $x \approx 0.3)$ can be seen from Fig. 4(c). As $\alpha_{\rho \max }$ is a measure of the electronic inhomogeneity of the films [20] [Fig. 4(a)], one can suppose it to be a measure of the cation disorder. A recent calculation suggests that the distribution of $\mathrm{La}$ and $\mathrm{Ca}$ cations modifies the elastic energy and affects the local electronic structure, stabilizing JT distortions [23]. Thus, for a given composition, cation distribution can fluctuate within an energy landscape from a random (solid-solution) configuration [5] to the atypical CE-type ( $\mathrm{La}, \mathrm{Ca}$ ) ordering shown here, with several local minima in between. Such an energy landscape provides a rational explanation to the variation of $T_{\mathrm{MI}}$ with $\alpha_{\rho \max }$ shown, and to the spatial distribution of inhomogeneity observed by STS for $D$ films. In disordered manganites, CMR is well explained based on phase separation scenario. On the other hand, the observed clean limit CMR in $O$ film [see Fig. 4(a)] can be interpreted as "CMR1" effect [24], involving a CE-AFM/FM phase boundary. The observed atypical CE-type ordering at the $A$ site brings about a similar order at the Mn site, resulting in a possible spin frustration in the form of AFM and FM chains [25], which appears important for the CMR [26]. Furthermore, CE-type charge modulation at Mn-sites has also been visualized by high resolution STM [27] above $T_{C}$.

Our experimental results allow formulating a common "superlattice" rule to search for the $A$-site ordered manganite films:

$$
\left[(m \mathrm{Ln}-\mathrm{Mn}-\mathrm{O}) /(2(\mathrm{Ln}-\mathrm{Mn}-\mathrm{B}-\mathrm{Mn}-\mathrm{O})]_{N}\right.
$$

with $m=0,1,2,3, \ldots ; \mathrm{Ln}=Y, \mathrm{La}, \mathrm{Nd}, \operatorname{Pr} \ldots$; and $B=$ $\mathrm{Ca}, \mathrm{Sr}, \mathrm{Ba}$. The ordered variants of LCMO films for different numbers of $\mathrm{LMO}$ layers " $m$ " are: $\mathrm{La}_{2 / 3} \mathrm{Ca}_{1 / 3} \mathrm{MnO}_{3}$ for $m=1 ; \mathrm{La}_{3 / 4} \mathrm{Ca}_{1 / 4} \mathrm{MnO}_{3}(m=2) ; \mathrm{La}_{4 / 5} \mathrm{Ca}_{1 / 5} \mathrm{MnO}_{3}(m=$ $3)$ and $\mathrm{La}_{5 / 6} \mathrm{Ca}_{1 / 6} \mathrm{MnO}_{3}(m=4)$. Such a growth strategy may provide a tool to verify whether the disorder-free manganite with a large average cation radius $(\mathrm{Ba})$ would possess a very high $T_{C}=530 \mathrm{~K}$ [5]. Finally, the interrelation between substrate misfit strain and $T_{\mathrm{MI}}$ has been previously explored $[28,29]$. But, our results indicate that the increase of the transition temperature and electronic homogeneity could be achieved intrinsically by controlling the structure and cation ordering for optimally doped manganite films with CMR effect.

The authors thank Ch. Renner for the help in STM/STS and E.Dagotto for fruitful discussion. Deutsche Forschungsgemeinschaft via SFB 602, TPA2 is acknowledged. L. S. thanks the Alexander von Humboldt Foundation.

[1] R. von Helmolt, J. Wecker, B. Holzapfel, L. Schultz, and K. Samwer, Phys. Rev. Lett. 71, 2331 (1993).

[2] H. Y. Hwang, S.-W. Cheong, P. G. Radaelli, M. Marezio, and B. Batlogg, Phys. Rev. Lett. 75, 914 (1995).

[3] P. Schiffer, A.P. Ramirez, W. Bao, and S.-W. Cheong, Phys. Rev. Lett. 75, 3336 (1995).

[4] A. J. Millis, Nature (London) 392, 147 (1998).

[5] L. M. Rodriguez and J.P. Attfield, Phys. Rev. B 54, R15 622 (1996).

[6] P. G. Radaelli, M. Marezio, H. Y. Hwang, S.-W. Cheong, and B. Batlogg, Phys. Rev. B 54, 8992 (1996).

[7] M. Uehara, S. Mori, C. H. Chen, and S.-W. Cheong, Nature (London) 399, 560 (1999).

[8] M. Fäth et al., Science 285, 1540 (1999).

[9] T. Becker et al., Phys. Rev. Lett. 89, 237203 (2002).

[10] Ch. Renner, G. Aeppli, B.-G. Kim, Y.-A. Soh, and S.-W. Cheong, Nature (London) 416, 518 (2002).

[11] E. Dagotto, T. Hotta, and A. Moreo, Phys. Rep. 344, 1 (2001).

[12] K. Ahn, T. Lookman, and A. R. Bishop, Nature (London) 428, 401 (2004).

[13] J. Burgy, M. Mayr, V. Martin-Mayor, A. Moreo, and E. Dagotto, Phys. Rev. Lett. 87, 277202 (2001).

[14] T. Arima et al., Phys. Rev. B 66, 140408(R) (2002).

[15] D. Akahoshi et al., Phys. Rev. Lett. 90, 177203 (2003).

[16] F. Millange et al., Chem. Mater. 10, 1974 (1998).

[17] V. Moshnyaga et al., Appl. Phys. Lett. 74, 2842 (1999).

[18] V. Moshnyaga et al., Nat. Mater. 2, 247 (2003).

[19] P. G. Radaelli, G. Iannone, M. Marezio, H. Y. Hwang, S.-W. Cheong, J.D. Jorgensen, and D. N. Argyriou, Phys. Rev. B 56, 8265 (1997).

[20] J. Mitra, M. Paranjape, A. K. Raychaudhuri, N. D. Mathur, and M. G. Blamire, Phys. Rev. B 71, 094426 (2005).

[21] V. Kiryukhin, T. Y. Koo, H. Ishibashi, J. P. Hill, and S.-W. Cheong Phys. Rev. B 67, 064421 (2003).

[22] J. W. Linn et al., Phys. Rev. Lett. 76, 4046 (1996).

[23] V. Ferrari, M. Towler, and P. B. Littlewood, Phys. Rev. Lett. 91, 227202 (2003).

[24] H. Aliaga et al., Phys. Rev. B 68, 104405 (2003).

[25] T. Hotta, A. Feiguin, and E. Dagotto, Phys. Rev. Lett. 86, 4922 (2001).

[26] R. Mathieu, D. Akahoshi, A. Asamitsu, Y. Tomioka, and Y. Tokura, Phys. Rev. Lett. 93, 227202 (2004).

[27] L. Sudheendra, V. Moshnyaga, B. Damaschke, and K. Samwer cond-mat/0605712.

[28] J. Y. Gu, K. H. Kim, T. W. Noh, and K.-S. Suh, J. Appl. Phys. 78, 6151 (1995).

[29] R. Shreekala et al., Appl. Phys. Lett. 74, 1886 (1999). 